\title{
Existence and Uniqueness of Positive Solutions for Singular Nonlinear Fractional Differential Equation via Mixed Monotone Operator Method
}

\author{
Tian Wang $\mathbb{D}^{1,2}$ and Zhaocai Hao $\mathbb{D}^{1,3}$ \\ ${ }^{1}$ School of Mathematical Sciences, Qufu Normal University, Qufu, 273165 Shandong, China \\ ${ }^{2}$ College of Applied Science, Beijing University of Technology, Beijing 100124, China \\ ${ }^{3}$ Department of Mathematics and Statistics, Missouri University of Science and Technology, Rolla, MO 65401, USA
}

Correspondence should be addressed to Zhaocai Hao; zchjal@163.com

Received 1 February 2020; Revised 9 March 2020; Accepted 18 March 2020; Published 10 July 2020

Guest Editor: Chuanjun Chen

Copyright ( 92020 Tian Wang and Zhaocai Hao. This is an open access article distributed under the Creative Commons Attribution License, which permits unrestricted use, distribution, and reproduction in any medium, provided the original work is properly cited.

In this article, we discuss the existence and uniqueness of positive solution for a class of singular fractional differential equations, where the nonlinear term contains fractional derivative and an operator. By applying the fixed point theorem in cone, we get the existence and uniqueness of positive solutions for the fractional differential equation. Moreover, we give an example to demonstrate our main result.

\section{Introduction}

In this article, we consider the existence and uniqueness of solutions for the following singular fractional differential equations:

$$
\left\{\begin{array}{l}
D_{0^{+}}^{\alpha} u(t)+p(t) f\left(t, u(t), D_{0+}^{\beta} u(t)\right)+q(t) g(t, u(t),(H u)(t))=0, \quad 0<t<1, \\
u(0)=u^{\prime}(0)=\cdots=u^{(n-2)}(0)=0 \\
{\left[D_{0^{+}}^{\gamma} u(t)\right]_{t=1}=k(u(1)),}
\end{array}\right.
$$

where $n-1<\alpha \leq n, n>3,1 \leq \beta \leq \gamma \leq n-2, p, q \in C((0,1)$, $[0, \infty)), p(t)$ and $q(t)$ are allowed to be singular at $t=0$ or $t=1, f:(0,1) \times(0, \infty) \times(0, \infty) \longrightarrow[0, \infty)$ is continuous and $f(t, u, v)$ may be singular at $t=0,1$ and $u=v=0, g$ : $(0,1) \times[0, \infty) \times[0, \infty) \longrightarrow[0, \infty)$ is continuous and $g(t$, $u, v)$ may be singular at $t=0,1$, and $k:[0, \infty) \longrightarrow[0, \infty)$ is continuous function.

In recent years, we can see that differential equations have more and more attention in many fields, like electrical networks, quantum physics, and probability. There are a lot of interesting results from fractional differential equation,
Schrodinger equation and k-hessian equations. In [1-4], the authors studied the solutions of fractional differential equations, and obtained some interesting results. Such as uniqueness of iterative positive solution, existence of multiple positive solutions or maximum and minimum solutions. In [5-9], the authors studied the solutions of Schrödinger equation. From these paper, there have several results about existence of infinitely solutions, existence and nonexistence of blow-up solutions, existence and asymptotic properties of solutions or existence and nonexistence of entire large solutions. In [10-12], the authors considered existence of blow-up radial solutions of the k-hessian equations or convergence analysis and uniqueness of blow-up solutions of the k-hessian equations. Inspired by these conclusions,we realize that the questions about differential equations are very interesting and varied. So we focus on fractional differential equation. The authors in [13] studied the following fractional differential equation:

$$
\left\{\begin{array}{l}
D_{0^{+}}^{\alpha} x(t)+f(t, x(t), x(t))+g(t, x(t))=0, \quad 0<t<1, \\
x^{(i)}(0)=0, \quad i=0,1,2, \cdots, n-2, \\
{\left[D_{0+}^{\beta} x(t)\right]_{t=1}=0, \quad 2 \leq \beta \leq n-2,}
\end{array}\right.
$$


where $n-1<\alpha \leq n, n>3, D_{0^{+}}^{\alpha}$ is the Riemann-Liouville fractional derivative, and $f:[0,1] \times[0, \infty) \times[0, \infty) \longrightarrow[0, \infty)$ is a continuous function.

In [14], by using cone expansion fixed point theorem, Li et al. studied the existence of positive solutions for the following fractional differential equation with RiemannStieltjes integral:

$$
\left\{\begin{array}{l}
D_{0^{+}}^{\alpha} x(t)+p(t) f(t, x(t))+q(t) g(t, x(t))=0, \quad 0<t<1, \\
x(0)=x^{\prime}(0)=\cdots=x^{(n-2)}(0)=0, \\
x(1)=\int_{0}^{1} k(s) x(s) d A(s),
\end{array}\right.
$$

where $n-1<\alpha \leq n, n \geq 2, p, q:(0,1) \longrightarrow[0, \infty)$ are continuous, $p, q \in L^{1}(0,1)$, and allowed to be singular at $t=0$ or $t=1, f, g:[0,1] \times(0, \infty) \longrightarrow[0, \infty)$ are continuous and $f(t, x), g(t, x)$ may be singular at $x=0, k:(0,1) \longrightarrow$ $[0, \infty)$ is continuous with $k \in L^{1}(0,1)$, where $\int_{0}^{1} k(s) x(s) d$ $A(s)$ is the Riemann-Stieltjes integral with a signed measure and $A:[0,1] \longrightarrow \mathbb{R}$ is a bounded variation function, and $D_{0^{+}}^{\alpha}$ is the standard Riemann-Liouville derivative of order $\alpha$.

In [15], Liu et al. investigated the iterative positive solutions for the following singular nonlinear fractional differential equation with integral boundary conditions.

$$
\left\{\begin{array}{l}
D_{0^{+}}^{\alpha} x(t)+p(t) f(t, x(t), x(t))+q(t) g(t, x(t))=0, \quad 0<t<1, \\
x(0)=x^{\prime}(0)=\cdots=x^{(n-2)}(0)=0, \\
x(1)=\int_{0}^{1} k(s) x(s) d A(s),
\end{array}\right.
$$

where $n-1<\alpha \leq n, n \geq 2, p, q:(0,1) \longrightarrow[0, \infty)$ are continuous and allowed to be singular at $t=0$ or $t=1, f:(0,1) \times$ $(0, \infty) \times(0, \infty) \longrightarrow[0, \infty)$ is continuous and $f(t, x, y)$ may be singular at $t=0,1$ and $x=y=0, g(t, x):(0,1) \times(0, \infty)$ $\longrightarrow[0, \infty)$ is continuous and may be singular at $t=0,1$ and $x=0, k:(0,1) \longrightarrow[0, \infty)$ is continuous with $k \in L^{1}$ $(0,1)$, where $\int_{0}^{1} k(s) x(s) d A(s)$ is the Riemann-Stieltjes integral with a signed measure and $A:[0,1] \longrightarrow \mathbb{R}$ is a bounded variation function, and $D_{0^{+}}^{\alpha}$ is the standard RiemannLiouville derivative of order $\alpha$.

In [16], Zhang and Tian considered the following fractional differential equation:

$$
\left\{\begin{array}{l}
D_{0^{+}}^{v} x(t)+f\left(t, x(t), D_{0+}^{\gamma} x(t)\right)+g(t, x(t))=0, \quad 0<t<1, \\
x^{(i)}(0)=0, \quad i=0,1,2, \cdots, n-2, \\
{\left[D_{0+}^{\beta} x(t)\right]_{t=1}=k(x(1))}
\end{array}\right.
$$

where $n-1<v<n, n>3,1 \leq \gamma \leq \beta \leq n-2$, and $f:[0,1] \times$ $[0, \infty) \times[0, \infty) \longrightarrow[0, \infty), \quad g:[0,1] \times[0, \infty) \longrightarrow[0, \infty)$, and $k:[0, \infty) \longrightarrow[0, \infty)$ are continuous functions.
Motivated by the abovementioned papers, the purpose of this article is to study the existence and uniqueness of positive solution for FBVP (1). Obviously, the problem in our article is more general. We not only consider the nonlinear term containing the derivative term, but also study the nonlinear term containing an operator. And we do not need the linearity of operator in nonlinear term. It may be a nonlinear operator.

Comparing with the results in [17], boundary value problem (1) has a more general form. First, we discuss the singularity. It means that $p, q$ are allowed to be singular at $t=0,1, f$ may be singular at $t=0,1$ and $x=y=0$, and $g$ may be singular at $t=0$. Second, we not only consider the derivative term but also consider the operator term, where the operator can be linear or nonlinear. Especially, when $p(t)=q(t)=1$ and $H u=0$, we can see that the problem in [16] is a special case of problem (1). Comparing with [13], firstly, when $k(u)=0$, $\beta=0, p(t)=q(t)=1$, and $H u(t)=u(t)$, then our problem (1) reduced compared to the problem in [13]. Third, in [13], the author did not consider the singularity, but in problem (1), we consider the singularity. Compared with [14], first, our method is different from that of [14]. We use the fixed point theorem for the sum of operators rather than the fixed point theorem of cone expansion and compression. Second, $f(t, x, y)$ not only has three variables but also is singular for both time and space variables, and $g(t, x, y)$ also has three variables. Compared with [15], we consider the derivative term and the operator term. Our results extended and improved some existing results, such as [13-16].

The rest of the paper is organized as follows: in Section 2, we give some preliminaries and lemmas that will be used in our main result. In Section 3, we study the existence and uniqueness of a positive solution of the BVP (1). In Section 4 , an example is provided to demonstrate our main results.

\section{Preliminaries and Lemmas}

In this section, we present some notations, definitions and lemmas that will be used in the paper.

Let $(E,\|\cdot\|)$ be a Banach space, and $\theta$ is defined as the zero element of $E$. A nonempty closed convex set $P \subset E$ is a cone if it satisfies (1) $x \in P, \lambda \geq 0 \Longrightarrow \lambda x \in P$ and (2) $x \in P$, $-x \in P \Longrightarrow x=\theta$. Putting $P^{\circ}=\{x \in P \mid x$ is an interior point of $P\}$, a cone $P$ is said to be solid if $P^{\circ}$ is nonempty. In this paper, suppose that $(E,\|\cdot\|)$ is a Banach space partially ordered by a cone $P \subset E$, that is, $x \leq y$ if and only if $y-x \in$ $P$. A cone $P$ called normal if there exists a constant $N>0$ such that for all $x, y \in E, \theta \leq x \leq y$ implies $\|x\| \leq N\|y\|$, and the smallest such $N$ is called the normality constant of $P$. For all $x, y \in P$, we denote the notation $x \sim y$ if there have constants $\lambda, \mu>0$ such that $\lambda y \leq x \leq \mu y$. Clearly, $\sim$ is an equivalence relation. Given $h>\theta$, we denote by $P_{h}$ the set $P_{h}=\{x \in E \mid x \sim h\}$. And it is easy to know that $P_{h} \subset P$.

Definition 1 (see [18]). An operator $A: P \times P \longrightarrow P$ is said to be a mixed monotone operator if $A(x, y)$ is increasing in the first component and decreasing in the second component, i.e., $u_{i}, v_{i}(i=1,2) \in P, u_{1} \leq u_{2}, v_{1} \geq v_{2}$, implies 
$A\left(u_{1}, v_{1}\right) \leq A\left(u_{2}, v_{2}\right)$. An element $x \in P$ is called a fixed point of $A$ if $A(x, x)=x$.

Definition 2 (see [19]). Let $D=P$ or $D=P^{\circ}$ and $\alpha$ be a real number with $0 \leq \alpha \leq 1$. An operator $A: D \longrightarrow D$ is said to be $\alpha$ concave if it satisfies

$$
A(t x) \geq t^{\alpha} A x, \quad \forall t \in(0,1), x \in D \text {. }
$$

Definition 3 (see [19]). An operator $B: P \longrightarrow P$ is said to be subhomogeneous if it satisfies

$$
B(t x) \geq t B x, \quad \forall t \in(0,1), x \in D .
$$

Definition 4 (see [20]). The Riemann-Liouville fractional integral of order $\alpha>0$ of a function $y:(0, \infty) \longrightarrow \mathbb{R}$ is given by

$$
I_{0^{+}}^{\alpha} y(t)=\frac{1}{\Gamma(\alpha)} \int_{0}^{t}(t-s)^{\alpha-1} y(s) d s
$$

provided that the right-hand side is pointwise defined on $(0, \infty)$.

Definition 5 (see [20]). The Riemann-Liouville fractional derivative of order $\alpha>0$ of a continuous function $y:(0, \infty)$ $\longrightarrow \mathbb{R}$ is given by

$$
D_{0^{+}}^{\alpha} y(t)=\frac{1}{\Gamma(n-\alpha)}\left(\frac{d}{d t}\right)^{n} \int_{0}^{t} \frac{y(s)}{(t-s)^{\alpha-n+1}} d s,
$$

where $n=[\alpha]+1$, where $[\alpha]$ denotes the integer part of the number $\alpha$, provided that the right-hand side is pointwise defined on $(0, \infty)$.

Lemma 6 (see [20]). Let $\alpha>0$. If we assume $u \in C(0,1) \cap$ $L^{1}(0,1)$, then the fractional differential equation

$$
D_{0^{+}}^{\alpha} u(t)=0
$$

has

$$
u(t)=C_{1} t^{\alpha-1}+C_{2} t^{\alpha-2}+\cdots+C_{N} t^{\alpha-N}, \quad C_{i} \in \mathbb{R}, i=1,2, \cdots, N,
$$

as the unique solution, where $N=[\alpha]+1$.

From the definition of the Riemann-Liouville derivative, we can obtain the statement.

Lemma 7 (see [20]). Assume that $u \in C(0,1) \cap L^{1}(0,1)$ with a fractional derivative of order $\alpha>0$ that belongs to $C(0,1) \cap$ $L^{1}(0,1)$. Then,

$$
I_{0^{+}}^{\alpha} D_{0^{+}}^{\alpha} u(t)=u(t)+C_{1} t^{\alpha-1}+C_{2} t^{\alpha-2}+\cdots+C_{N} t^{\alpha-N},
$$

for some $C_{i} \in \mathbb{R}(i=1,2, \cdots, N)$, where $N=[\alpha]+1$.
In the following, we present the Green's function of FBVP (1).

Lemma 8 (see [16]). Let $h \in C[0,1]$, then the unique solution of the linear problem

$$
\left\{\begin{array}{l}
D_{0^{+}}^{\alpha} u(t)+h(t)=0, \quad 0<t<1, n-1<\alpha \leq n, \\
u(0)=u^{\prime}(0)=\cdots=u^{(n-2)}(0)=0, \\
{\left[D_{0^{+}}^{\gamma} u(t)\right]_{t=1}=k(u(1)), \quad 1 \leq \gamma \leq n-2}
\end{array}\right.
$$

is given by

$$
u(t)=\int_{0}^{1} G(t, s) h(s) d s+\frac{\Gamma(\alpha-\gamma)}{\Gamma(\alpha)} k(u(1)) t^{\alpha-1},
$$

where

$G(t, s)=\frac{1}{\Gamma(\alpha)}\left\{\begin{array}{l}t^{\alpha-1}(1-s)^{\alpha-\gamma-1}-(t-s)^{\alpha-1}, \quad 0 \leq s \leq t \leq 1 \\ t^{\alpha-1}(1-s)^{\alpha-\gamma-1}, \quad 0 \leq t \leq s \leq 1\end{array}\right.$

Lemma 9 (see [16]). The Green's function $G(t, s)$ defined by (15) satisfies the following properties:

(1) $G:[0,1] \times[0,1] \longrightarrow[0, \infty)$ is continuous

(2) For any $t, s \in[0,1]$, there is

$$
\begin{aligned}
0 & \leq t^{\alpha-1}(1-s)^{\alpha-\gamma-1}\left[1-(1-s)^{\gamma}\right] \\
& \leq \Gamma(\alpha) G(t, s) \leq t^{\alpha-1}(1-s)^{\alpha-\gamma-1}
\end{aligned}
$$

(3) For any $t, s \in[0,1]$, there is

$$
\begin{aligned}
0 & \leq t^{\alpha-\beta-1}(1-s)^{\alpha-\gamma-1}\left[1-(1-s)^{\gamma-\beta}\right] \\
& \leq \Gamma(\alpha-\beta) D_{0+}^{\beta} G(t, s) \leq t^{\alpha-\beta-1}(1-s)^{\alpha-\gamma-1}
\end{aligned}
$$

In [17], the operator equation $A(x, x)+B(x, x)=x$ was studied, where $A, B$ are mixed monotone operators, and for all $t \in(0,1), \psi(t) \in(t, 1]$, there have $A\left(t x, t^{-1} y\right) \geq \psi(t)$ $A(x, y), B\left(t x, t^{-1} y\right) \geq t B(x, y)$. Based on the result in [17], we get the following lemma:

Lemma 10. Let $P$ be a normal cone in $E$, and let $A, B: P \times$ $P \longrightarrow P$ be two mixed monotone operators and $C: P \longrightarrow P$ is a decreasing operator, suppose that

(A1) for all $t \in(0,1)$, there have $\psi(t) \in(t, 1]$ such that

$$
A\left(t x, t^{-1} y\right) \geq \psi(t) A(x, y), \quad \text { for all } x, y \in P
$$

(A2) for all $t \in(0,1)$,

$$
B\left(t x, t^{-1} y\right) \geq t B(x, y), \quad \text { for all } x, y \in P
$$


(A3) for all $t \in(0,1)$,

$$
C\left(t^{-1} y\right) \geq t C(y), \quad \text { for all } y \in P
$$

(A4) there exists $h>\theta$ and $h \in P_{h}$, such that $A(h, h) \in$ $P_{h}, B(h, h) \in P_{h}, C(h) \in P_{h}$

(A5) there exists a constants $\delta>0$, such that for all $x$, $y \in P$,

$$
A(x, y) \geq \delta(B(x, y)+C(y))
$$

Then

(1) $A: P_{h} \times P_{h} \longrightarrow P_{h}, B: P_{h} \times P_{h} \longrightarrow P_{h}, C: P_{h} \longrightarrow P_{h}$

(2) There exist $u_{0}, v_{0} \in P_{h}$ and $r \in(0,1)$ such that

$$
\begin{aligned}
r v_{0} & \leq u_{0}<v_{0}, u_{0} \leq A\left(u_{0}, v_{0}\right)+B\left(u_{0}, v_{0}\right)+C v_{0} \\
& \leq A\left(v_{0}, u_{0}\right)+B\left(v_{0}, u_{0}\right)+C u_{0} \leq v_{0}
\end{aligned}
$$

(3) The operator equation $A(x, x)+B(x, x)+C x=x$ has a unique solution $x^{*}$ in $P_{h}$

(4) For any initial values $x_{0}, y_{0} \in P_{h}$, constructing successively the sequences

$$
\begin{array}{ll}
x_{n}=A\left(x_{n-1}, y_{n-1}\right)+B\left(x_{n-1}, y_{n-1}\right)+C y_{n-1}, & n=1,2, \cdots, \\
y_{n}=A\left(y_{n-1}, x_{n-1}\right)+B\left(y_{n-1}, x_{n-1}\right)+C x_{n-1}, & n=1,2, \cdots,
\end{array}
$$

we have $x_{n} \longrightarrow x^{*}$ and $y_{n} \longrightarrow x^{*}$ as $n \longrightarrow \infty$

Proof. Now we define the operator $T: P \times P \longrightarrow P$ by

$$
T(x, y)=A(x, y)+B(x, y)+C y, \quad x, y \in P .
$$

So it is easy to know that $T$ is a mixed monotone operator. Next, we will prove that $T(h, h) \in P_{h}$ and $T\left(t x, t^{-1} y\right) \geq$ $\varphi(t) T(x, y)$, where $\varphi(t) \in(t, 1]$, Then according to the results of [21], the conclusions of Lemma 10 holds.

Firstly, we will show that $T(h, h) \in P_{h}$ for $h>\theta$ and $h \in P_{h}$. From (18)-(20), for any $t \in(0,1), x, y \in P$, there have

$$
\begin{gathered}
A\left(t^{-1} x, t y\right) \leq(\psi(t))^{-1} A(x, y), \\
B\left(t^{-1} x, t y\right) \leq t^{-1} B(x, y), \\
C(t y) \leq t^{-1} C y .
\end{gathered}
$$

According to (A4), we may assume that there exist constants $\mu_{i}>0, v_{i}>0(i=1,2,3)$ such that

$$
\begin{aligned}
& \mu_{1} h \leq A(h, h) \leq v_{1} h, \\
& \mu_{2} h \leq B(h, h) \leq v_{2} h, \\
& \mu_{3} h \leq C h \leq v_{3} h .
\end{aligned}
$$

Choose sufficiently small number $a \in(0,1)$ such that $a h$ $\leq x, y \leq(1 / a) h$ for any $x, y \in P_{h}$; then from (18)-(20) and (25)-(28), there have

$$
\begin{aligned}
& A(x, y) \leq A\left(\frac{1}{a} h, a h\right) \leq \frac{1}{\psi(a)} A(h, h) \leq \frac{1}{\psi(a)} v_{1} h, \\
& A(x, y) \geq A\left(a h, \frac{1}{a} h\right) \geq \psi(a) A(h, h) \geq \psi(a) \mu_{1} h,
\end{aligned}
$$

and we know $(1 /(\psi(a))) v_{1}, \psi(a) \mu_{1}>0$. So $A(x, y) \in P_{h}$ and $A: P_{h} \times P_{h} \longrightarrow P_{h}$. Similarly, we can easily get that $B(x, y)$ $\in P_{h}, B: P_{h} \times P_{h} \longrightarrow P_{h}, C(y) \in P_{h}$, and $C: P_{h} \longrightarrow P_{h}$. Then, we have $T(h, h)=A(h, h)+B(h, h)+C h \in P_{h}$.

Second, we will prove that $T\left(t x, t^{-1} y\right) \geq \varphi(t) T(x, y)$ is satisfied for any $x, y \in P, t \in(0,1), \varphi(t) \in(t, 1]$.

For any $x, y \in P$, from (A5), we have

$$
A(x, y)+\delta A(x, y) \geq \delta(B(x, y)+C y)+\delta A(x, y)
$$
$y)$. So

Then $(1+\delta) A(x, y) \geq \delta(A(x, y)+B(x, y)+C y)=\delta T(x$,

$$
A(x, y) \geq \frac{T(x, y)}{1+\delta^{-1}}
$$

Thus, there have

$$
\begin{aligned}
T\left(t x, t^{-1} y\right)-t T(x, y)= & A\left(t x, t^{-1} y\right)+B\left(t x, t^{-1} y\right)+C\left(t^{-1} y\right) \\
& -t(A(x, y)+B(x, y)+C y) \\
\geq & (\psi(t)-t) A(x, y)+B\left(t x, t^{-1} y\right) \\
& -t B(x, y)+C\left(t^{-1} y\right)-t C y \\
\geq & (\psi(t)-t) A(x, y) \geq \frac{\psi(t)-t}{1+\delta^{-1}} T(x, y) .
\end{aligned}
$$

So

$$
T\left(t x, t^{-1} y\right) \geq\left(t+\frac{\psi(t)-t}{1+\delta^{-1}}\right) T(x, y) .
$$

Now we note $\varphi(t)=t+\left((\psi(t)-t) /\left(1+\delta^{-1}\right)\right)$; it is easy to know that $\varphi(t) \in(t, 1]$, where $t \in(0,1)$. Then, $T\left(t x, t^{-1} y\right) \geq$ $\varphi(t) T(x, y)$.

Thus, by the results of Zhai and Zhang in [21], we know what the conclusions of Lemma 10 hold. 


\section{Main Results}

For convenience, in this section, we set

$$
E=\left\{x \mid x \in C[0,1], D_{0+}^{\beta} x \in C[0,1]\right\}
$$

Clearly, $E$ is a Banach space with the norm

$$
x=\max \left\{\max _{t \in[0,1]}|x(t)|, \max _{t \in[0,1]}\left|D_{0+}^{\beta} x(t)\right|\right\} .
$$

Now, we let the cone $P$ be defined by

$$
P=\left\{x \in E \mid x(t) \geq 0, D_{0+}^{\beta} x(t) \geq 0, \forall t \in[0,1]\right\}
$$

There have $P \subset E$, and $P$ is a normal cone. And, the order relation in $E$ is given by $x \leq y$ if $x(t) \leq y(t), D_{0+}^{\beta} x(t) \leq D_{0+}^{\beta} y(t)$.

From Lemma 8 , we get that the unique solution of the problem (1) satisfies the following equation:

$$
\begin{aligned}
x(t)= & \int_{0}^{1} G(t, s) p(s) f\left(s, x(s), D_{0+}^{\beta} x(s)\right) d s \\
& +\int_{0}^{1} G(t, s) q(s) g(s, x(s),(H x)(s)) d s \\
& +\frac{\Gamma(\alpha-\gamma)}{\Gamma(\alpha)} k(x(1)) t^{\alpha-1},
\end{aligned}
$$

where $G(t, s)$ is the Green's function given in (15).

Theorem 11. Assume that the following assumptions hold:

(H1) $p, q:(0,1) \longrightarrow[0, \infty)$ are continuous, and $p(t), q$ ( $t)$ are allowed to be singular at $t=0$ or $t=1$. For all $t \in(0,1)$, there is $p(t) \geq q(t) \geq m$, where $m$ is a constant

(H2) $f:(0,1) \times(0, \infty) \times(0, \infty) \longrightarrow[0, \infty), g:(0,1) \times$ $[0, \infty) \times[0, \infty) \longrightarrow[0, \infty), k:(0,1) \longrightarrow[0, \infty)$ are continuous, $f(t, u, v)$ may be singular at $t=0,1$, and $u=v=0, g(t, u, v)$, may be singular at $t=0,1$

(H3) For fixed $t \in(0,1), v \in(0, \infty), f(t, u, v)$ is increasing in $u \in(0, \infty)$ and decreasing in $v \in(0, \infty)$; for fixed $t \in(0,1), v \in(0, \infty), g(t, u, v)$ is increasing in $u \in$ $(0, \infty)$ and decreasing in $v \in(0, \infty)$; for fixed $t$ $\in(0,1), k(v)$ is decreasing in $v \in(0, \infty)$ with $k$ $(v(1)) \neq 0$

(H4) There exists a constant $\vartheta \in(0,1)$ such that for all $\eta, t \in(0,1)$ and $u, v \in(0, \infty)$,

$$
\begin{aligned}
f\left(t, \eta u, \eta^{-1} v\right) & \geq \eta^{9} f(t, u, v), \\
g\left(t, \eta u, \eta^{-1} v\right) & \geq \eta g(t, u, v), \\
k\left(\eta^{-1} v\right) & \geq \eta k(v)
\end{aligned}
$$

(H5) $g(s, 1,1) \equiv 0$. There exists a constant $\delta_{1}>0, \delta_{2}>$ 0 such that for all $t \in(0,1)$ and $u, v \in(0, \infty), f$ $(t, u, v) \geq \delta_{1} g(t, u, 0), f(t, u, v) \geq \delta_{2} k(v)$

(H6) $\int_{0}^{1}(1-s)^{\alpha-\gamma-1} p(s) s^{9(1-\alpha)} f(s, 1,1) d s<\infty$ and $\int_{0}^{1}$ $(1-s)^{\alpha-1} q(s) s^{1-\alpha} g(s, 1,1) d s<\infty$

(H7) $H: C[0,1] \longrightarrow C[0,1] . H u \geq 0$ and $H u$ is increasing in $u \in P$. For $\lambda \in(0,1)$ and $u \in P$, there is $H(\lambda u) \geq$ $\lambda \mathrm{Hu}$

Then problem (1) has a unique positive solution $u^{*} \in P_{h}$, where $h(t)=t^{\alpha-1}$. For any initial value $u_{0}, v_{0} \in P_{h}$, there are two iterative sequences $\left\{u_{n}\right\},\left\{v_{n}\right\}$ for approximating $u^{*}, v^{*}$, that is, $u_{n} \longrightarrow u^{*}, v_{n} \longrightarrow v^{*}$, as $n \longrightarrow \infty$, where

$$
\begin{aligned}
u_{n}(t)= & \int_{0}^{1} G(t, s) f\left(s, u_{n-1}(s), D_{0+}^{\beta} v_{n-1}(s)\right) d s \\
& +\int_{0}^{1} G(t, s) g\left(s, u_{n-1}(s), H v_{n-1}(s)\right) d s \\
& +\frac{\Gamma(\alpha-\gamma)}{\Gamma(\alpha)} k\left(v_{n-1}(1)\right) t^{\alpha-1}, \quad n=1,2, \cdots, \\
v_{n}(t)= & \int_{0}^{1} G(t, s) f\left(s, v_{n-1}(s), D_{0+}^{\beta} u_{n-1}(s)\right) d s \\
& +\int_{0}^{1} G(t, s) g\left(s, v_{n-1}(s), H u_{n-1}(s)\right) d s \\
& +\frac{\Gamma(\alpha-\gamma)}{\Gamma(\alpha)} k\left(u_{n-1}(1)\right) t^{\alpha-1}, \quad n=1,2, \cdots .
\end{aligned}
$$

Proof. We define operators $A, B, C$ as follows:

$$
A(u, v)(t)=\int_{0}^{1} G(t, s) p(s) f\left(s, u(s), D_{0+}^{\beta} v(s)\right) d s, \quad t \in(0,1)
$$

$$
\begin{aligned}
B(u, v)(t) & =\int_{0}^{1} G(t, s) q(s) g(s, u(s), H v(s)) d s, \quad t \in(0,1) \\
C v(t) & =\frac{\Gamma(\alpha-\gamma)}{\Gamma(\alpha)} k(v(1)) t^{\alpha-1}, \quad t \in(0,1)
\end{aligned}
$$

Furthermore, for $u, v \in P$, we obtain that

$$
\begin{aligned}
D_{0+}^{\beta} A(u, v)(t)= & \int_{0}^{1} D_{0+}^{\beta} G(t, s) p(s) f \\
& \cdot\left(s, u(s), D_{0+}^{\beta} v(s)\right) d s, \quad S S t \in(0,1), \\
D_{0+}^{\beta} B(u, v)(t)= & \int_{0}^{1} D_{0+}^{\beta} G(t, s) q(s) g \\
& \cdot(s, u(s), H v(s)) d s, \quad t \in(0,1), \\
D_{0+}^{\beta} C v(t)= & \frac{\Gamma(\alpha-\gamma)}{\Gamma(\alpha-\beta)} k(v(1)) t^{\alpha-\beta-1}, \quad t \in(0,1) .
\end{aligned}
$$


Clearly, $u$ is the solution of problem (1) if and only if $u=A(u, u)+B(u, u)+C u$. So, if we can prove that the operators $A, B, C$ satisfy all conditions in Lemma 10 , then we will obtain the conclusions in Theorem 11.

First, we will prove that $A, B$ are well defined. Evidently, $C$ is well defined, so we omit it.

From (H4), for all $t \in(0,1), \eta \in(0,1), u, v \in(0, \infty)$, we have

$$
\begin{aligned}
& f(t, u, v)=f\left(t, \eta \eta^{-1} u, \eta^{-1} \eta v\right) \geq \eta^{9} f\left(t, \eta^{-1} u, \eta v\right), \\
& g(t, u, v)=g\left(t, \eta \eta^{-1} u, \eta^{-1} \eta v\right) \geq \eta g(t, \eta-1 u, \eta v) .
\end{aligned}
$$

Furthermore, we have

$$
\begin{aligned}
& f\left(t, \eta^{-1} u, \eta v\right) \leq \eta^{-9} f(t, u, v), \\
& g\left(t, \eta^{-1} u, \eta v\right) \leq \eta^{-1} g(t, u, v) .
\end{aligned}
$$

Taking $u=v=1$ in (H4) and (47), for $\eta \in(0,1), t \in(0,1)$, we have

$$
\begin{aligned}
& f\left(t, \eta, \eta^{-1}\right) \geq \eta^{9} f(t, 1,1), \\
& f\left(t, \eta^{-1}, \eta\right) \leq \eta^{-9} f(t, 1,1), \\
& g\left(t, \eta, \eta^{-1}\right) \geq \eta g(t, 1,1), \\
& g\left(t, \eta^{-1}, \eta\right) \leq \eta^{-1} g(t, 1,1) .
\end{aligned}
$$

For any $u, v \in P_{h}$, there exists a constant $b>0$ such that $b h \leq u, v \leq(1 / b) h$, where $h(t)=t^{\alpha-1}, t \in(0,1)$. So, from the above inequalities, we obtain

$$
\begin{aligned}
f(t, u(t), v(t)) & \leq f\left(t, \frac{1}{b} t^{\alpha-1}, b t^{\alpha-1}\right) \leq f\left(t, \frac{1}{b} t^{1-\alpha}, b t^{\alpha-1}\right) \\
& \leq t^{9(1-\alpha)} f\left(t, \frac{1}{b}, b\right) \leq t^{9(1-\alpha)}\left(\frac{1}{b}\right)^{9} f(t, 1,1), \\
f(t, u(t), v(t)) & \geq f\left(t, b t^{\alpha-1}, \frac{1}{b} t^{\alpha-1}\right) \geq f\left(t, b t^{\alpha-1}, \frac{1}{b} t^{1-\alpha}\right) \\
& \geq t^{9(\alpha-1)} f\left(t, b, \frac{1}{b}\right) \geq t^{9(\alpha-1)} b^{9} f(t, 1,1), \\
g(t, u(t), v(t)) & \leq g\left(t, \frac{1}{b} t^{\alpha-1}, b t^{\alpha-1}\right) \leq g\left(t, \frac{1}{b} t^{1-\alpha}, b t^{\alpha-1}\right) \\
& \leq t^{(1-\alpha)} g\left(t, \frac{1}{b}, b\right) \leq t^{(1-\alpha)}\left(\frac{1}{b}\right) g(t, 1,1), \\
g(t, u(t), v(t)) & \geq g\left(t, b t^{\alpha-1}, \frac{1}{b} t^{\alpha-1}\right) \geq g\left(t, b t^{\alpha-1}, \frac{1}{b} t^{1-\alpha}\right) \\
& \geq t^{(\alpha-1)} g\left(t, b, \frac{1}{b}\right) \geq t^{(\alpha-1)} b g(t, 1,1) .
\end{aligned}
$$

Then, combining assumption (H6), we get

$$
\begin{aligned}
& \int_{0}^{1} G(t, s) p(s) f\left(s, u(s), D_{0+}^{\beta} v(s)\right) d s \\
& \leq \int_{0}^{1} G(t, s) p(s) s^{9(1-\alpha)}\left(\frac{1}{b}\right)^{\vartheta} f(s, 1,1) d s \\
& \leq \frac{t^{\alpha-1}}{\Gamma(\alpha)} \int_{0}^{1}(1-s)^{\alpha-\gamma-1} p(s) s^{9(1-\alpha)} \\
& \cdot\left(\frac{1}{b}\right)^{\vartheta} f(s, 1,1) d s<\infty,
\end{aligned}
$$

$$
\begin{aligned}
& \int_{0}^{1} D_{0+}^{\beta} G(t, s) p(s) f\left(s, u(s), D_{0+}^{\beta} v(s)\right) d s \\
& \leq \int_{0}^{1} D_{0+}^{\beta} G(t, s) p(s) s^{9(1-\alpha)}\left(\frac{1}{b}\right)^{\vartheta} f(s, 1,1) d s \\
& \leq \frac{t^{\alpha-\beta-1}(1-s)^{\alpha-\gamma-1}}{\Gamma(\alpha-\beta)} \int_{0}^{1}(1-s)^{\alpha-\gamma-1} p(s) s^{9(1-\alpha)} \\
& \cdot\left(\frac{1}{b}\right)^{9} f(s, 1,1) d s<\infty,
\end{aligned}
$$

$$
\begin{aligned}
& \int_{0}^{1} G(t, s) q(s) g(s, u(s), H v(s)) d s \\
& \leq \int_{0}^{1} G(t, s) q(s) s^{1-\alpha} \frac{1}{b} g(s, 1,1) d s \\
& \leq \frac{t^{\alpha-1}}{\Gamma(\alpha)} \int_{0}^{1}(1-s)^{\alpha-\gamma-1} q(s) s^{1-\alpha} \frac{1}{b} g \\
& \quad \cdot(s, 1,1) d s<\infty,
\end{aligned}
$$

$$
\begin{gathered}
\int_{0}^{1} D_{0+}^{\beta} G(t, s) q(s) g(s, u(s), H v(s)) d s \\
\leq \int_{0}^{1} D_{0+}^{\beta} G(t, s) q(s) s^{1-\alpha} \frac{1}{b} g(s, 1,1) d s \\
\leq \frac{t^{\alpha-\beta-1}(1-s)^{\alpha-\gamma-1}}{\Gamma(\alpha-\beta)} \int_{0}^{1}(1-s)^{\alpha-\gamma-1} q \\
\cdot(s) s^{1-\alpha} \frac{1}{b} g(s, 1,1) d s<\infty .
\end{gathered}
$$

Thus, we know that $A, B$ are well defined.

Second, we will prove that $A, B, C$ satisfy conditions (A1)-(A3) of Lemma 10. From (H1)-(H3), for all $u, v \in$ $P, t \in(0,1)$, we have $A(u, v)(t) \geq 0, D_{0+}^{\beta} A(u, v)(t) \geq 0, B(u$, $v)(t) \geq 0, D_{0+}^{\beta} B(u, v)(t) \geq 0, C v(t) \geq 0, C v(t) \geq 0$. Therefore, $A: P \times P \longrightarrow P, B: P \times P \longrightarrow P, C: P \times P \longrightarrow P$. And for all $u, v \in P, t \in(0,1), u \leq v$ means $u(t) \leq v(t), D_{0+}^{\beta} u(t) \leq D_{0+}^{\beta} v$ $(t)$. Thus, from assumptions $(\mathrm{H} 2)$ and $(\mathrm{H} 3)$, it is clear that operators $A, B, C$ satisfy conditions (A1)-(A3) of Lemma 10. And $A, B$ are mixed monotone operators and $C$ is a decreasing operator. 
Third, we show that $A(h, h) \in P_{h}, B(h, h) \in P_{h}, C(h) \in P_{h}$. From (H2) and (H3) and Lemma 9, we have

$$
\begin{aligned}
& A(h, h)=\int_{0}^{1} G(t, s) p(s) f\left(s, s^{\alpha-1}, D_{0+}^{\beta} s^{\alpha-1}\right) d s \\
& \leq \int_{0}^{1} \frac{t^{\alpha-1}(1-s)^{\alpha-\gamma-1}}{\Gamma(\alpha)} p(s) s^{9(1-\alpha)}\left(\frac{1}{b}\right)^{\vartheta} f(s, 1,1) d s \\
& =h(t) \int_{0}^{1} \frac{(1-s)^{\alpha-\gamma-1}}{\Gamma(\alpha)} p(s) s^{9(1-\alpha)}\left(\frac{1}{b}\right)^{\vartheta} f(s, 1,1) d s, \\
& A(h, h)=\int_{0}^{1} G(t, s) p(s) f\left(s, s^{\alpha-1}, D_{0+}^{\beta} s^{\alpha-1}\right) d s \\
& \geq \int_{0}^{1} \frac{t^{\alpha-1}(1-s)^{\alpha-\gamma-1}\left[1-(1-s)^{\gamma}\right]}{\Gamma(\alpha)} \\
& \cdot p(s) s^{9(\alpha-1)} b^{9} f(s, 1,1) d s \\
& =h(t) \int_{0}^{1} \frac{(1-s)^{\alpha-\gamma-1}\left[1-(1-s)^{\gamma}\right]}{\Gamma(\alpha)} \\
& \cdot p(s) s^{9(\alpha-1)} b^{9} f(s, 1,1) d s, \\
& D_{0+}^{\beta} A(h, h)=\int_{0}^{1} D_{0+}^{\beta} G(t, s) p(s) f\left(s, s^{\alpha-1}, D_{0+}^{\beta} s^{\alpha-1}\right) d s \\
& \leq \int_{0}^{1} \frac{t^{\alpha-\beta-1}(1-s)^{\alpha-\gamma-1}}{\Gamma(\alpha-\beta)} p(s) s^{9(1-\alpha)}\left(\frac{1}{b}\right)^{\vartheta} f(s, 1,1) d s \\
& =D_{0+}^{\beta} h(t) \int_{0}^{1} \frac{(1-s)^{\alpha-\gamma-1}}{\Gamma(\alpha)} p(s) s^{\vartheta(1-\alpha)} \\
& \cdot\left(\frac{1}{b}\right)^{\vartheta} f(s, 1,1) d s \text {, } \\
& D_{0+}^{\beta} A(h, h)=\int_{0}^{1} D_{0+}^{\beta} G(t, s) p(s) f\left(s, s^{\alpha-1}, D_{0+}^{\beta} s^{\alpha-1}\right) d s \\
& \geq \int_{0}^{1} \frac{t^{\alpha-1}(1-s)^{\alpha-\gamma-1}\left[1-(1-s)^{\gamma-\beta}\right]}{\Gamma(\alpha)} \\
& \text { - } p(s) s^{9(\alpha-1)} b^{9} f(s, 1,1) d s \\
& =D_{0+}^{\beta} h(t) \int_{0}^{1}\left(\frac{(1-s)^{\alpha-\gamma-1}\left(1-(1-s)^{\gamma-\beta}\right)}{\Gamma(\alpha)} p(s)\right) \\
& \cdot s^{9(\alpha-1)} b^{9} f(s, 1,1) d s \text {. }
\end{aligned}
$$

Set

$$
\begin{aligned}
c_{1} & =\int_{0}^{1} \frac{(1-s)^{\alpha-\gamma-1}\left[1-(1-s)^{\gamma-\beta}\right]}{\Gamma(\alpha)} p(s) s^{9(\alpha-1)} b^{9} f(s, 1,1) d s, c_{2} \\
& =\int_{0}^{1} \frac{(1-s)^{\alpha-\gamma-1}}{\Gamma(\alpha)} p(s) s^{9(1-\alpha)}\left(\frac{1}{b}\right)^{\vartheta} f(s, 1,1) d s .
\end{aligned}
$$

Now, in a similar way, for all $t \in(0,1)$, we obtain that

$$
\begin{aligned}
B(h, h) \leq & h(t) \int_{0}^{1} \frac{(1-s)^{\alpha-\gamma-1}}{\Gamma(\alpha)} q(s) s^{1-\alpha} \frac{1}{b} g(s, 1,1) d s, \\
B(h, h) \geq & h(t) \int_{0}^{1} \frac{(1-s)^{\alpha-\gamma-1}\left[1-(1-s)^{\gamma}\right]}{\Gamma(\alpha)} \\
& \cdot q(s) s^{\alpha-1} b g(s, 1,1) d s, \\
D_{0+}^{\beta} B(h, h) \leq & D_{0+}^{\beta} h(t) \int_{0}^{1} \frac{(1-s)^{\alpha-\gamma-1}}{\Gamma(\alpha)} q(s) s^{1-\alpha} \frac{1}{b} g(s, 1,1) d s, \\
D_{0+}^{\beta} B(h, h) \geq & D_{0+}^{\beta} h(t) \int_{0}^{1} \frac{(1-s)^{\alpha-\gamma-1}\left[1-(1-s)^{\gamma-\beta}\right]}{\Gamma(\alpha)} \\
& \cdot q(s) s^{\alpha-1} b g(s, 1,1) d s .
\end{aligned}
$$

Set

$$
\begin{aligned}
& c_{3}=\int_{0}^{1} \frac{(1-s)^{\alpha-\gamma-1}\left[1-(1-s)^{\gamma-\beta}\right]}{\Gamma(\alpha)} q(s) s^{\alpha-1} b g(s, 1,1) d s, \\
& c_{4}=\int_{0}^{1} \frac{(1-s)^{\alpha-\gamma-1}}{\Gamma(\alpha)} q(s) s^{1-\alpha} \frac{1}{b} g(s, 1,1) d s .
\end{aligned}
$$

Because of $g(t, 1,1) \equiv 0$, obviously, $c_{4} \geq c_{3}>0$. Then $c_{3} h \preceq B(h, h) \leq c_{4} h$. And we have

$$
s^{9(\alpha-1)} b^{9} f(s, 1,1) \geq s^{\alpha-1} b f(s, 1,1) \geq s^{\alpha-1} b \delta_{1} g(s, 1,1) .
$$

Combining with the condition $p(t) \geq q(t)$, it is clear that $0<\delta_{1} c_{3} \geq c_{1} \leq c_{2}$. So, there is $c_{1} h \leq A(h, h) \leq c_{2} h$. And from $k(v(1)) \neq 0$ and (42) and (45), obviously, we obtain that $C h \in P_{h}$.

Finally, we show that operators $A, B, C$ satisfy assumption (A5) of Lemma 10.

According to assumption (H6), for $t \in(0,1)$, we know $h(t) \in(0,1)$. So $0 \geq H h \geq H 1$. From assumption (H5), for all $t \in(0,1), u, v \in P_{h}$, there is

$$
\begin{aligned}
A(u, v) & =\int_{0}^{1} G(t, s) p(s) f\left(s, u(s), D_{0+}^{\beta} v(s)\right) d s \\
& \geq \int_{0}^{1} G(t, s) q(s) \delta_{1} g(s, u(s), 0) d s \\
& \geq \int_{0}^{1} G(t, s) q(s) \delta_{1} g(s, u(s), H v(s)) d s \\
& =\delta_{1} B(u, v),
\end{aligned}
$$




$$
\begin{aligned}
D_{0+}^{\beta} A(u, v) & =\int_{0}^{1} D_{0+}^{\beta} G(t, s) p(s) f\left(s, u(s), D_{0+}^{\beta} v(s)\right) d s \\
& \geq \int_{0}^{1} D_{0+}^{\beta} G(t, s) q(s) \delta_{1} g(s, u(s), 0) d s \\
& \geq \int_{0}^{1} D_{0+}^{\beta} G(t, s) q(s) \delta_{1} g(s, u(s), H v(s)) d s \\
& =\delta_{1} D_{0+}^{\beta} B(u, v) .
\end{aligned}
$$

Then, $A(u, v) \preceq \delta_{1} B(u, v)$.

$$
\begin{aligned}
A(u, v) & =\int_{0}^{1} G(t, s) p(s) f\left(s, u(s), D_{0+}^{\beta} v(s)\right) d s \\
& \geq \int_{0}^{1} G(t, s) m \delta_{2} d s \geq m \delta_{2} \frac{t^{\alpha-1}}{\Gamma(\alpha)}\left(\frac{1}{\alpha-\gamma}-\frac{1}{\alpha}\right) \\
& \geq m k(v(1)) \frac{t^{\alpha-1}}{\Gamma(\alpha)}\left(\frac{1}{\alpha-\gamma}-\frac{1}{\alpha-\beta}\right) \\
& =\frac{m}{\Gamma(\alpha-\gamma)}\left(\frac{1}{\alpha-\gamma}-\frac{1}{\alpha-\beta}\right) C v,
\end{aligned}
$$

$$
\begin{aligned}
D_{0+}^{\beta} A(u, v) & =\int_{0}^{1} D_{0+}^{\beta} G(t, s) p(s) f\left(s, u(s), D_{0+}^{\beta} v(s)\right) d s \\
& \geq \int_{0}^{1} D_{0+}^{\beta} G(t, s) m \delta_{2} k(v(1)) d s \\
& \geq m \delta_{2} k(v(1)) \frac{t^{\alpha-\beta-1}}{\Gamma(\alpha-\beta)}\left(\frac{1}{\alpha-\gamma}-\frac{1}{\alpha-\beta}\right) \\
& =\frac{m \delta_{2}}{\Gamma(\alpha-\beta)}\left(\frac{1}{\alpha-\gamma}-\frac{1}{\alpha-\beta}\right) D_{0+}^{\beta} C v .
\end{aligned}
$$

Then, $A(u, v) \geq \delta^{\prime} C v$, where $\delta^{\prime}=\left(m \delta_{2} /(\Gamma(\alpha-\beta))\right)((1 /$ $(\alpha-\gamma))-(1 /(\alpha-\beta)))$. Now, choose $\delta=\min \left\{\delta_{1}, \delta^{\prime}\right\}$, then $A(u, v) \geq \delta(B(u, v)+C v)$.

So, applying Lemma 10 , the proof of Theorem 11 is completed.

Remark 12. In Lemma 10, if we assume that $A, B: P_{h} \times P_{h}$ $\longrightarrow P_{h}$ and $C: P_{h} \times P_{h}$, then it is easy to prove the results in Lemma 10 are also satisfied. So, we also can use the changed lemma to prove Theorem 11.

\section{Example}

Example 13. We consider the following FBVP:

$$
\left\{\begin{array}{l}
D_{0^{+}}^{9 / 2} u(t)+\frac{2}{t}\left(t^{2}+\sqrt{u(t)}\right)+\frac{1}{t \sqrt{D_{0+}^{3 / 2} u(t)+1}}+\frac{1}{t} \int_{0}^{t} u(t) d t=0, \quad 0<t<1, \\
u(0)=u^{\prime}(0)=u^{\prime \prime}(0)=u^{\prime \prime \prime}(0)=0 \\
{\left[D_{0+}^{5 / 2} u(t)\right]_{t=1}=\frac{1}{\sqrt{u(1)+1}} .}
\end{array}\right.
$$

Let $p(t)=q(t)=1 / t, f(t, u, v)=t^{2}+\sqrt{u}+(1 / \sqrt{v+1}), g$ $(t, u, v)=t^{2}+\sqrt{u}+v, k(v)=1 / \sqrt{v+1}$. Clearly, $p, q, f, g, k$ satisfied conditions (H1)-(H3). And for $H u(t)=\int_{0}^{t} u(t) d t$, we can see that assumption (H7) is satisfied. For $\eta \in(0,1)$, we have

$$
\begin{aligned}
f\left(t, \eta u, \eta^{-1} v\right) & =t^{2}+\sqrt{\eta u}+\frac{1}{\sqrt{\eta^{-1} v+1}}=t^{2} \sqrt{\eta u}+\frac{\sqrt{\eta}}{\sqrt{v+\eta}} \\
& \geq \sqrt{\eta}\left(t^{2}+\sqrt{u}+\frac{1}{\sqrt{v+1}}\right)=\eta^{1 / 2} f(t, u, v), \\
g\left(t, \eta u, \eta^{-1} v\right) & =t^{2}+\sqrt{\eta u}+\eta^{-1} v \geq \eta t^{2}+\eta \sqrt{u}+\eta v \\
& =\eta\left(t^{2}+\sqrt{u}+v\right)=\eta g(t, u, v), \\
k\left(\eta^{-1} v\right) & =\frac{1}{\sqrt{\eta^{-1} v+1}} \geq \eta \frac{1}{\sqrt{v+1}}=\eta k(v) .
\end{aligned}
$$

Then, we know that assumption (H4) of Theorem 11 is satisfied.
Clearly, $g(t, 1,1) \equiv 0$. Moreover, if we let $\delta_{1}=\delta_{2}=1$, then we have

$f(t, u, v)=t^{2}+\sqrt{u}+\frac{1}{\sqrt{v+1}} \geq t^{2}+\sqrt{u}=g(t, u, 0)$,

$f(t, u, v)=t^{2}+\sqrt{u}+\frac{1}{\sqrt{v+1}} \geq \frac{1}{\sqrt{v+1}}=k(v)$.

Then, assumption (H5) of Theorem 11 is satisfied.

Finally, we show that assumption (H6) of Theorem 11 is also satisfied. It is easy to see from the following inequalities:

$$
\begin{aligned}
& \int_{0}^{1}(1-s)^{\alpha-\gamma-1} p(s) s^{9(1-\alpha)} f(s, 1,1) d s \\
& \quad=\int_{0}^{1}(1-s) s^{-1} s^{-(7 / 4)}\left(s^{2}+1+\frac{\sqrt{2}}{2}\right)<\infty,
\end{aligned}
$$




$$
\begin{aligned}
& \int_{0}^{1}(1-s)^{\alpha-1} q(s) s^{1-\alpha} g(s, 1,1) d s \\
& \quad=\int_{0}^{1}(1-s)^{(7 / 4)} s^{-1} s^{-(7 / 4)}\left(s^{2}+1+s\right) d s<\infty .
\end{aligned}
$$

Therefore the assumptions of Theorem 11 are satisfied. Thus, problem (58) possesses the uniqueness solution.

Remark 14. It is clear that $H u(t)=\int_{0}^{1} u(t) d t$ is linear in this example. If we let operator $H$ be a nonlinear operator, for example, $H u(t)=u^{a}(t)$, where $0<a<1$, it is obvious that assumption (H7) of Theorem 11 is also satisfied. So using different operators, we also can obtain that BVP (58) has a unique positive solution from the same discussion process. This fact means that our theorem has a wide range of applications.

\section{Data Availability}

No data were used to support this study.

\section{Conflicts of Interest}

The authors declare that they have no conflicts of interest.

\section{Acknowledgments}

The authors would like to thank the referees and the Editor for their careful reading and their important comments and helpful suggestions on improving the presentation of this paper.

\section{References}

[1] X. Zhang, L. Liu, and Y. Wu, "Multiple positive solutions of a singular fractional differential equation with negatively perturbed term," Mathematical and Computer Modelling, vol. 55, no. 3-4, pp. 1263-1274, 2012.

[2] L. Guo, L. Liu, and Y. Wu, "Uniqueness of iterative positive solutions for the singular fractional differential equations with integral boundary conditions," Boundary Value Problems, vol. 2016, 2016.

[3] T. Ren, S. Li, X. Zhang, and L. Liu, "Maximum and minimum solutions for a nonlocal p-Laplacian fractional differential system from eco-economical processes," Boundary Value Problems, vol. 2017, no. 1, 2017.

[4] J. Tan, C. Cheng, and X. Zhang, "Positive solutions of fractional differential equation nonlocal boundary value problems," Advances in Difference Equations, vol. 2015, no. 1, 2015.

[5] X. Zhang, L. Liu, Y. Wu, and Y. Cui, "Existence of infinitely solutions for a modified nonlinear Schrödinger equation via dual approach," Electronic Journal of Differential Equations, vol. 2018, no. 147, pp. 1-15, 2018.

[6] X. Zhang, Y. Wu, and Y. Cui, "Existence and nonexistence of blow-up solutions for a Schrödinger equation involving a nonlinear operator," Applied Mathematics Letters, vol. 82, pp. 85-91, 2018.
[7] X. Zhang, J. Jiang, Y. Wu, and Y. Cui, "Existence and asymptotic properties of solutions for a nonlinear Schrödinger elliptic equation from geophysical fluid flows," Applied Mathematics Letters, vol. 90, pp. 229-237, 2019.

[8] X. Zhang, J. Jiang, Y. Wu, and Y. Cui, "The existence and nonexistence of entire large solutions for a quasilinear Schrödinger elliptic system by dual approach," Applied Mathematics Letters, vol. 100, article 106018, 2020.

[9] X. Zhang, L. Liu, Y. Wu, and Y. Cui, "The existence and nonexistence of entire large solutions for a quasilinear Schrödinger elliptic system by dual approach," Journal of Mathematical Analysis and Applications, vol. 464, no. 2, pp. 1089-1106, 2018.

[10] X. Zhang, L. Liu, Y. Wu, and Y. Cui, "A sufficient and necessary condition of existence of blow-up radial solutions for a $k$ -Hessian equation with a nonlinear operator," Nonlinear Analysis: Modelling and Control, vol. 25, pp. 126-143, 2020.

[11] X. Zhang, J. Xu, J. Jiang, Y. Wu, and Y. Cui, "The convergence analysis and uniqueness of blow-up solutions for a Dirichlet problem of the general $k$-hessian equations," Applied Mathematics Letters, vol. 102, article 106124, 2020.

[12] J. He, X. Zhang, L. Liu, and Y. Wu, "Existence and nonexistence of radial solutions of the Dirichlet problem for a class of general k-hessian equations," Nonlinear Analysis: Modelling and Control, vol. 23, no. 4, pp. 475-492, 2018.

[13] M. Jleli and B. Samet, "Existence of positive solutions to an arbitrary order fractional differential equation via a mixed monotone operator method," Nonlinear Analysis: Modelling and Control, vol. 20, no. 3, pp. 367-376, 2015.

[14] H. Li, L. Liu, and Y. Wu, "Positive solutions for singular nonlinear fractional differential equation with integral boundary conditions," Boundary Value Problems, vol. 2015, no. 1, 2015.

[15] L. L. Liu, X. Zhang, L. Liu, and Y. Wu, "Iterative positive solutions for singular nonlinear fractional differential equation with integral boundary conditions," Advances in Difference Equations, vol. 2016, no. 1, 2016.

[16] L. Zhang and H. Tian, "Existence and uniqueness of positive solutions for a class of nonlinear fractional differential equations," Advances in Difference Equations, vol. 2017, no. 1, 2017.

[17] L. Liu, X. Zhang, J. Jiang, and Y. Wu, "The unique solution of a class of sum mixed monotone operator equations and its application to fractional boundary value problems," Journal of Nonlinear Sciences and Applications, vol. 9, no. 5, pp. 2943-2958, 2016.

[18] D. Guo and V. Lakshmikantham, Nonlinear Problems in Abstract Cones, Academic Press, Boston, MA, USA, 1988.

[19] H. Wang and L. Zhang, "The solution for a class of sum operator equation and its application to fractional differential equation boundary value problems," Boundary Value Problems, vol. 2015, no. 1, 2015.

[20] A. A. Kilbas, H. M. Srivastava, and J. J. Trujillo, Theory and Applications of Fractional Differential Equations, Elsevier B. V, Netherlands, 2006.

[21] C. Zhai and L. Zhang, "New fixed point theorems for mixed monotone operators and local existence-uniqueness of positive solutions for nonlinear boundary value problems," Journal of Mathematical Analysis and Applications, vol. 382, no. 2, pp. 594-614, 2011. 